\section{Differential roles of TGF- $\beta$ signalling in joint tissues during osteoarthritis development}

Osteoarthritis (OA) is the most common degenerative joint disease. The aetiology of $\mathrm{OA}$ is multifactorial, including joint injury, obesity, ageing and heredity. Inactivation of transforming growth factor beta (TGF- $\beta$ ) or its downstream molecules may be an important signalling event contributing to OA pathogenesis because mutations of Smad3, a central molecule in TGF- $\beta$ signalling, have been found in patients with early-onset OA. ${ }^{1-3}$ It has been known for many years that TGF- $\beta$ promotes matrix protein synthesis, inhibits chondrocyte hypertrophy and stimulates expression of Prg4, which encodes for lubricin, a critical molecule for lessening joint friction. ${ }^{4}$ Lubricin, which is present in synovial fluid and on the surface (superficial layer) of articular cartilage, plays an important role in joint lubrication and synovial homeostasis.

The disease of OA affects the entire joint. ${ }^{5}$ TGF- $\beta$ signalling may play differential roles in joint tissues during OA development. For example, Smad3 global knockout (KO) mice showed inhibition of chondrocyte hypertrophy and OA-like articular cartilage damage. ${ }^{6}$ The deletion of Tgfbr 2 , encoding for type II TGF- $\beta$ receptor, ${ }^{7}$ or $\operatorname{Smad} 3^{8}$ in articular chondrocytes consistently led to an OA-like phenotype. In contrast, the activation of TGF- $\beta$ signalling in mesenchymal progenitor cells of subchondral bone also caused OA-like lesions. ${ }^{9}$ These findings suggest that TGF- $\beta$ signalling may play differential roles in the various joint tissues.

A recent report in the Annals of the Rheumatic Diseases demonstrated that the disruption of decorin-restricted TGF- $\beta$ signalling led to increased articular cartilage matrix stiffness, rendering joints more resistant to OA. ${ }^{10}$ This suggests that the loss of Dcn could decelerate OA development because of the release of endogenous TGF- $\beta$ into the joint. To further understand the functional significance of decorin in regulation of TGF- $\beta$ signalling and in OA development, it will be important to generate $D c n$ conditional $\mathrm{KO}$ mice targeting different tissues in the joint. It will also be interesting to determine whether mutation(s) of $D c n$ can be detected in patients with an aneurysms-OA syndrome in which Smad3 has been proposed as a disease-causing gene for some patients.

Ting Yu Wang, ${ }^{1}$ Di Chen ${ }^{2}$

${ }^{1}$ Department of Pharmacy, Shanghai Ninth People's Hospital, Shanghai Jiao Tong University School of Medicine, Shanghai, China
${ }^{2}$ Department of Biochemistry, Rush University Medical Center, Chicago, Illinois, USA

Correspondence to Di Chen, Department of Biochemistry, Rush University Medical Center, Chicago, IL 60612, USA; di_chen@rush.edu

Contributors TYW: concept and writing; DC: concept and revising.

Funding National Nature Science Foundation of China (grant nos. 81301531, 81572104).

Competing interests None declared.

Patient consent Obtained.

Provenance and peer review Not commissioned; internally peer reviewed.

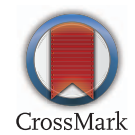

To cite Wang TY, Chen D. Ann Rheum Dis 2016;75:e72.

Accepted 4 August 2016

Published Online First 19 August 2016

Ann Rheum Dis 2016;75:e72. doi:10.1136/annrheumdis-2016-210312

\section{REFERENCES}

1 Valdes AM, Spector TD, Tamm A, et al. Genetic variation in the Smad3 gene is associated with hip and knee osteoarthritis. Arthritis Rheum 2010;62:2347-52.

2 van de Laar IM, Oldenburg RA, Pals G, et al. Mutations in SMAD3 cause a syndromic form of aortic aneurysms and dissections with early-onset osteoarthritis. Nat Genet 2011:43:121-6.

3 van de Laar IM, van der Linde D, Oei EH, et al. Phenotypic spectrum of the Smad3-related aneurysms-osteoarthritis syndrome. J Med Genet 2012;49:47-57.

4 Cuellar A, Reddi AH. Stimulation of Superficial Zone Protein/Lubricin/PRG4 by Transforming Growth Factor- $\beta$ in Superficial Zone Articular Chondrocytes and Modulation by Glycosaminoglycans. Tissue Eng Part A 2015;21:1973-81.

5 Loeser RF, Goldring SR, Scanzello CR, et al. Osteoarthritis: a disease of the joint as an organ. Arthritis Rheum 2012;64:1697-707.

6 Yang X, Chen L, Xu X, et al. TGF- $\beta / S$ mad3 signals repress chondrocyte hypertrophic differentiation and are required for maintaining articular cartilage. J Cell Biol 2001;153:35-46.

7 Shen J, Li J, Wang B, et al. Deletion of the transforming growth factor $\beta$ receptor type II gene in articular chondrocytes leads to a progressive osteoarthritis-like phenotype in mice. Arthritis Rheum 2013;65:3107-19.

8 Chen CG, Thuillier D, Chin EN, et al. Chondrocyte-intrinsic Smad3 represses Runx2-inducible matrix metalloproteinase 13 expression to maintain articular cartilage and prevent osteoarthritis. Arthritis Rheum 2012;64:3278-89.

9 Zhen $G$, Wen $C$, Jia $X$, et al. Inhibition of TGF- $\beta$ signaling in mesenchymal stem cells of subchondral bone attenuates osteoarthritis. Nat Med 2013:19:704-12.

10 Gronau T, Krüger K, Prein C, et al. Forced exercise-induced osteoarthritis is attenuated in mice lacking the small leucine-rich proteoglycan decorin. Ann Rheum Dis 2016; Published Online First 4 July 2016. 